Revue d'histoire de l'Amérique française

THEVUE D.HISTOIRE DE L'AMÉRIQUE FRANÇAISE

\title{
La régionalisation de la production à l'Office national du film du Canada
}

\section{Pierre Verronneau}

Volume 55, numéro 4, printemps 2002

URI : https://id.erudit.org/iderudit/010440ar

DOI : https://doi.org/10.7202/010440ar

Aller au sommaire du numéro

Éditeur(s)

Institut d'histoire de l'Amérique française

ISSN

0035-2357 (imprimé)

1492-1383 (numérique)

Découvrir la revue

Citer cet article

Verronneau, P. (2002). La régionalisation de la production à l'Office national du film du Canada. Revue d'histoire de l'Amérique française, 55(4), 507-537.

https://doi.org/10.7202/010440ar
Résumé de l'article

Organisme d'État, l'Office national du film du Canada a fonctionné de manière centralisée de sa création en 1939 jusqu'au début des années 1970. C'est alors que le gouvernement central décide d'établir une politique du film pour le pays et annonce que la régionalisation de la production fait partie de ses priorités. Il fait ainsi écho aux voix régionales qui revendiquaient une telle décision. Le texte étudie la mise en place de la production régionale francophone (Acadie, Ontario, Ouest) dans le contexte social et politique du Québec et du Canada. Il aborde les contradictions entre les revendications nationales québécoises et celles des francophones hors Québec. Il expose les réactions et les résistances provenant des différentes instances du siège social de Montréal. Il propose enfin une remise en perspective de la production française de l'ONF des trente dernières années à la lumière de ce volet spécifique de son histoire. 


\title{
La régionalisation de la production à l'Office national du film du Canada
}

\author{
PIERRE VÉRONNEAU \\ Cinémathèque québécoise
}

RÉSUMÉ - Organisme d'État, l'Office national du film du Canada a fonctionné de manière centralisée de sa création en 1939 jusqu'au début des années 1970. C'est alors que le gouvernement central décide d'établir une politique du film pour le pays et annonce que la régionalisation de la production fait partie de ses priorités. II fait ainsi écho aux voix régionales qui revendiquaient une telle décision. Le texte étudie la mise en place de la production régionale francophone (Acadie, Ontario, Ouest) dans le contexte social et politique du Québec et du Canada. II aborde les contradictions entre les revendications nationales québécoises et celles des francophones hors Québec. II expose les réactions et les résistances provenant des différentes instances du siège social de Montréal. II propose enfin une remise en perspective de la production française de l'ONF des trente dernières années à la lumière de ce volet spécifique de son histoire.

Aвstract - As a State-owned institution, the National Film Board of Canada had a centralized management from its creation in 1939 to the early 1970s. Then the Federal Government adopted a film policy for the whole nation and announced that the regionalization of production was part of its priorities. By doing so, it echoed regional voices demanding such a decision. This article analyses the setting up of the French speaking regional production (in Acadia, Ontario, and the West) within the social and political context then prevailing in Quebec and Canada. It examines the contradictions between Quebec's national claims and those of the Francophones outside Quebec. It sheds light on the reaction and resistance expressed by various people in Montreal head office. And finally it proposes a rethinking about NFB's French production during the last thirty years from the point of view of that specific part of its history. 


\section{L}

E TRAVAIL DE L'HISTORIEN du cinéma, tel que nous le concevons, pose esthétiques, qu'elle s'inscrit toujours dans un contexte social, culturel et politique, et que les représentations qu'elle convie apportent, du point de vue historique, une certaine connaissance des sociétés, de leurs institutions et de leurs valeurs. En outre l'histoire du cinéma ne se limite pas aux œuvres, mais doit également prendre en compte les institutions qui gouvernent leur production, leur distribution, leur exploitation et leur réception ainsi que les discours produits sur le champ cinématographique ${ }^{1}$. Dans la majorité des cas, le film n'est pas le fruit d'une génération spontanée, mais découle d'un processus où s'expriment la volonté d'un créateur, les attentes d'une institution ou les pressions des circonstances et de la société. Dans le cas d'un organisme d'État comme l'Office national du film du Canada (ONF), s'ajoutent des déterminations de nature politique au sens organisationnel du terme ${ }^{2}$.

1. Il faut noter par ailleurs qu'au sein de la discipline historique, de plus en plus d'historiens, en Europe et en Amérique, s'inscrivent dans une problématique que l'on connaît sous la notion d' "histoire et cinéma ». On les retrouve au sein d'associations comme l'International Association for Media and History et de revues comme Film and History qu'éditent le Historians Film Committee, Film-Historia, Historical Journal of Film, Radio and Television, Journal of Popular Culture, Journal of Popular Film and Television, Film History et, à l'occasion, Journal of American History, Vingtième siècle et Sociétés et représentations. Le lecteur pourra aussi consulter Marc Ferro, Cinéma et histoire (Paris, Denoël-Gonthier, 1977), Robert C. Allen et Douglas Gomery, Film History. Theory and Practice (Montréal, McGraw-Hill, 1985), Michèle Lagny, De l'histoire du cinéma. Méthode historique et histoire du cinéma (Paris, Armand Colin, 1992), Antoine de Baecque et Christian Delage, De l'histoire au cinéma (Bruxelles, Éditions complexe, 1998). Il existe aussi de nombreux ouvrages plus spécifiques. Sur le terrain des rapports entre histoire et cinéma, les approches varient mais toujours il s'agit d'envisager les films comme facteurs et témoins de l'histoire, et d'étudier leur production, leur distribution et leur réception à la lumière des méthodes de la discipline historique et dans le contexte de l'histoire sociale et culturelle. Le cinéma est autant objet de l'histoire que l'histoire est elle-même objet de cinéma. À l'intérieur de ce large spectre, il n'est pas surprenant que les méthodes et les approches soient fort disparates et qu'il soit difficile de les catégoriser.

2. Mentionnons que l'ONF est administré par un conseil nommé par le gouvernement et dirigé par un commissaire à la cinématographie. Ce dernier est assisté de directeurs (production, distribution, services techniques) de qui relèvent les producteurs, les chefs de service et, en dernière instance, les cinéastes, les techniciens et le personnel administratif et de soutien. Le gouvernement (ou le ministre de tutelle) n'intervient pas directement dans la gestion de l'organisme (le fameux arm's length), mais détermine des orientations par les budgets et les lois qu'il vote, les programmes qu'il lance, les politiques qu'il établit. C'est au conseil d'administration et aux cadres supérieurs d'en tirer les conséquences concrètes. Si certains films répondent directement aux besoins des ministères, plusieurs émargent au programme général (un en français, un en anglais) défini par la production et les cinéastes. Les choix et arbitrages reviennent au Comité du programme en fonction des budgets de chaque studio (leur nombre et objectifs varient au fil des ans). 
Dans le cadre de cet article, nous tenterons de rendre le plus intégralement possible les mouvements pluriels et les nombreuses contradictions qui ont entouré et porté l'émergence et le développement de la production régionale onéfienne et de les inscrire dans les contextes sociopolitiques québécois et canadiens ${ }^{3}$. Cette histoire n'a fait l'objet, à ce jour, d'aucune étude approfondie. Pire, elle a été occultée à l'intérieur de l'histoire générale de l'ONF et, dans le cas de la production francophone, son appréciation a été subordonnée au point de vue québécois. L'objectif de cette étude sera donc de jeter une lumière nouvelle sur la production régionale onéfienne. Il ne sera pas question des films comme tels - ce serait un tout autre sujet et une autre manière d'aborder la relation cinéma et histoire — mais plutôt des institutions qui en assurent la gestion et des instances qui les commandent. Nous verrons qu'à l'intérieur de l'appareil d'État qu'est l'ONF, les dynamiques sont complexes et que les acteurs peuvent poursuivre des buts contradictoires. En outre l'ensemble est en perpétuelle relation avec des dynamiques externes, gouvernementales ${ }^{4}$ (fédérales et provinciales) ou sociales. Leur mise au jour permettra de voir comment la question de la régionalisation de la production à l'ONF constitue une manifestation des autres affirmations régionales dans les domaines politiques et culturels.

\section{LE TOURNAGE EN RÉGION}

Pour comprendre l'histoire de cette production, il faut rappeler l'origine du tournage dans ces zones géographiques situées hors des grands centres et qu’on dénomme régions. De mai 1939, date de fondation de l’ONF par

3. Nous avons mené la recherche préparatoire à la rédaction de ce texte à la Médiathèque Guy-L. Coté et aux Archives des Collections afférentes au film de la Cinémathèque québécoise, mais surtout au Service des archives de l'Office national du film à Montréal (grâce à la collaboration de son responsable Bernard Lutz). Nous avons eu accès à de nombreuses sources premières internes (notes de services, documents de planification, rapports, comptes rendus de réunion, procès-verbaux du Comité du programme, mémoires, etc.) et externes (rapports annuels, communiqués de presse, rapports gouvernementaux, etc.), sans parler des sources journalistiques ou publiées, ni des rencontres avec certains acteurs de cette histoire. Nous avons enfin bénéficié de l'appui du Studio documentaire Acadie/Moncton (dirigé alors par Diane Poitras). Cette recherche s'inscrit dans le prolongement de la publication de mon ouvrage Résistance et affirmation : la production francophone à l'ONF - 1939-1964 (Histoire du cinéma au Québec III) (Montréal, Cinémathèque québécoise, 1987).

4. Par exemple, sur le terrain spécifique du cinéma, les commissions royales d'enquête, les énoncés de politiques cinématographiques et culturelles, l'existence d'un autre organisme d'État chargé d'investir dans la production privée (la Société de développement de l'industrie cinématographique canadienne, aujourd'hui Téléfilm Canada) sans parler des relations avec RadioCanada. 
le gouvernement du Canada, jusqu'à 1956, le siège social de l'organisme est situé à Ottawa et les équipes de réalisation doivent se déplacer vers les provinces pour tourner les films prévus au programme général. Le centre prend l'habitude de couvrir les régions par son propre rayonnement et de correspondre de ce fait à toutes les parties. Dans le contexte du travail de la Commission royale d'enquête sur l'avancement des Arts, des Lettres et des Sciences au Canada, devant la nécessité d'adapter la société d'État à la réalité d'après-guerre et dans la foulée du rapport commandé à la firme J. D. Woods \& Gordon 5 , le gouvernement modifie la loi qui régit l'organisme. Celle-ci stipule notamment que l'ONF doit "produire et distribuer des films destinés à faire connaître et comprendre le Canada aux Canadiens et aux autres nations ${ }^{6} \%$. Malgré une production inégale des sujets régionaux, les films que l'on pourrait qualifier de «reportages pancanadiens» tirent très bien leur épingle du jeu, surtout avec l'avènement, en 1952, de la télévision, grâce à laquelle l'ONF jouira dorénavant de fenêtres de diffusion hebdomadaires. Le mouvement est analogue tant du côté anglophone que du côté francophone, mais il va assez vite se spécifier et prendre des envergures différentes. Le programme de régionalisation aura un impact beaucoup plus important au Canada anglais ${ }^{7}$. Pour éviter une certaine confusion dans le cadre de cet article, nous parlerons dorénavant uniquement de la production francophone. Quand l'ONF déménage son siège social à Montréal à l'été de $1956^{8}$, sa dynamique se modifie. On recrutera dorénavant davantage de cinéastes québécois et ceux-ci accepteront de se rendre plus aisément en région, ce qui veut dire en pratique hors de Montréal mais toujours au Québec.

Au début des années 1960, la dynamique centre/périphérie qui marque toute l'histoire canadienne connaît un nouveau développement à la faveur du réveil national et régional et des luttes nationalistes qui s'expriment au Québec. Sur plusieurs terrains (économique, politique, social), des tensions

5. The National Film Board: Survey of Organization and Business Administration (mars 1950).

6. Loi relative à l'Office national du film (Imprimeur de la Reine, 1950), article 9.

7. Voir le chapitre «Film Board Regionalization: Will the Centre Hold?», dans D. B. Jones, Movies and Memoranda. An Interpretative History of the National Film Board of Canada (Ottawa, Canadian Film Institute, 1981), 176-196.

8. L'ONF avait besoin d'un bâtiment unique et adapté où tous ses services de production seraient regroupés. Puisqu'il s'agit d'un organisme de création, le choix se pose entre les deux capitales culturelles du Canada : Montréal et Toronto. Le gouvernement choisit Montréal pour affirmer le caractère biculturel de l'organisme et attirer des Canadiens français, ceux-ci ne pouvant trouver à Toronto un cadre qui favorise leur créativité. Les terrains sont expropriés au printemps de 1953. 
se développent entre les intérêts nationaux et les intérêts provinciaux, sinon plus locaux ${ }^{9}$. Le gouvernement canadien se retrouve partagé entre des positions contradictoires. D'une part, il défend un concept national centralisateur mais, d'autre part, il doit composer avec l'affirmation de régionalismes divers, souvent économiques, parfois politiques et culturels, dont il tient d'ailleurs compte ${ }^{10}$. Parmi ceux-ci, le régionalisme québécois lui cause davantage de soucis, car il est mâtiné d'une saveur indépendantiste qui risque de lui laisser un mauvais goût dans la bouche. Une des manières pour le neutraliser sera de favoriser l'expression d'autres régionalismes francophones, quitte à jouer un peu avec le feu et à attiser les sentiments nationalistes québécois et acadiens ${ }^{11}$. Mais revenons à l'ONF pour voir comme cela se traduit dans la réalité.

Pendant les années 1960, en même temps que le nationalisme souffle sur le Québec, une volonté d'affirmation d'autonomie frappe la production francophone. Cela aboutit, en 1964, à la création d'une Production française autonome, parallèle à la Production anglaise. On y retrouve un certain nombre de studios dirigés chacun par un producteur. Cette production française est dominée par les Québécois. Très rares sont ceux qui proviennent de l'extérieur de la province. Il n'est pas étonnant dès lors que cette production réponde aux besoins et aux attentes du Québec. Les luttes nationalistes débordent aussi sur le champ sémantique. À la même époque, de même qu'on assiste au Québec à un rejet de l'appellation "Canadien français», remplacée par «Québécois», le cinéma francophone produit par l'ONF devient uniquement du cinéma "québécois». Il ne peut plus être canadienfrançais. On constate donc que, dans le vocabulaire, les membres d'une institution nationale canadienne excluent par conséquent une partie de la population qui parle leur langue, celle des francophones hors Québec, qui pourrait souhaiter exprimer sa culture par l'entremise du cinéma, mais qui ne peut se définir comme "québécoise». Conséquemment, sur ce terrain, il va se créer une dynamique centre-région particulière autant sur le terri-

9. Voir par exemple le traitement particulier réservé à la Gaspésie et au Bas-Saint-Laurent avec la mise sur pied du Bureau d'aménagement de l'Est du Québec qui engendrera autant l'enthousiasme que la déception.

10. Cela se reflète par exemple autant dans la création d'une Agence de développement régional que dans la création d'un ministère de l'Expansion économique régionale qui doit voir à l'amenuisement des disparités régionales.

11. Un film acadien, Robichaud (Herménégilde Chiasson, 1989) évoque d'ailleurs cette période. Le réalisateur veut montrer l'importance historique de l'homme dont l'apport à la modernisation du Canada n'est pas moindre à ses yeux que celui de Jean Lesage. Le réveil des Acadiens et l'affirmation de leur identité datent de cette époque. 
toire du Québec que sur l'ensemble du Canada d'expression française ${ }^{12}$. Une partie du cinéma onéfien se tournera donc en région mais par des cinéastes qui proviennent du centre. L'ONF n'est porté à développer ni une production régionale québécoise ni une production hors Québec. Toutefois, il encourage d'une certaine manière les sujets régionaux soit par des films au regard touristico-ethnologique, comme c'est le cas depuis sa fondation, soit par la reprise du régionalisme à l'intérieur d'un discours d'auteur (on peut penser à Pierre Perrault ${ }^{13}$ ).

Mais une autre tendance voit le jour dans la seconde moitié des années 1960, tout à fait dans l'esprit qui anime les sciences sociales de l'époque, les groupes populaires et le cinéma d'intervention politique : il s'agit de donner aux gens des régions la parole et l'image, de définir le cinéma selon leurs prémisses, et de se servir du film comme outil d'animation sociale. À cette époque, tant le gouvernement du Québec que celui du Canada manifestent un certain intérêt pour la région, le premier grâce au Bureau d'aménagement de l'Est du Québec (BAEQ), le second en s'intéressant aux problèmes des régions rurales. C'est à la suite d'un programme fédéral-provincial que naît le projet ARDA (Aménagement rural et développement agricole) doté d'un volet cinématographique. On veut utiliser le film documentaire comme instrument de changement et de sensibilisation. Par ailleurs, dans la foulée de son programme de lutte à la pauvreté, le gouvernement canadien crée en 1967 le programme interministériel «Challenge for Change» qui vise la promotion des personnes, des minorités et des masses ainsi que l'éveil de leur prise de conscience - et la prise en main de leurs propres affaires - grâce aux communications modernes. Du côté francophone, ce programme prend en 1969 le nom de «Société nouvelle». Bien que sous l'entière responsabilité de l'ONF, ce programme autonome n'émarge pas directement à son budget, ce qui lui confère une plus grande autonomie ${ }^{14}$.

12. Pour comprendre cet apparent paradoxe, il faut bien voir que le siège social étant situé au Québec, plusieurs représentants de l'ONF ne verront pas la nécessité de définir le Québec comme une région et réserveront cette appellation aux collectivités situées hors Québec. Toutefois, comme du point de vue québécois ce qui est éloigné de la région métropolitaine accède au statut de région, on assistera à des revendications «extérieures», provenant par exemple du Saguenay, de l'Abitibi ou du Bas Saint-Laurent, ce qui va amener l'ONF à employer le mot «région» pour désigner les deux réalités, québécoises et hors Québec. On en viendra même à créer à Québec un studio régional "Québec» pour bien marquer la distinction avec le siège social montréalais, qui jouit d'un statut et d'un mandat national canadien.

13. Mentionnons son cycle sur l'île aux Coudres.

14. Ce programme, au budget duquel l'ONF contribue pour la moitié, prend fin en 1980 par manque de fonds; l'intérêt sociopolitique gouvernemental n'y est plus. L'ONF en a publié un 
On voit poindre alors dans le prolongement de «Société nouvelle» un nouvel enjeu : doit-on donner à la population, particulièrement celle qui vit loin du centre montréalais, des moyens de production cinématographique ou lui envoyer des professionnels qui répondraient à ses besoins? L'ONF tranche de manière significative. Il estime qu'il n'est pas essentiel, pour poursuivre ce genre d'activités, de disperser ses structures de production, bien implantées dans son siège social de Montréal où se retrouve la quasi-totalité du personnel. Il défend donc la compétence exclusive du centre dans une activité de nature professionnelle, le cinéma, et refuse la décentralisation. Seuls seront acceptés les projets qui proviennent de cinéastes originaires des régions, mais travaillant déjà au sein de l'ONF. Tel est le cas de l'Acadien Léonard Forest qui tourne, entre 1967 et 1972, plusieurs films originaux à sujets acadiens ${ }^{15}$. À la lumière de cette expérience liée à "Société nouvelle», Forest devient de plus en plus convaincu de la nécessité de créer en Acadie un studio de production, condition à ses yeux essentielle à la présence de films acadiens au programme de l'ONF. Mais les dirigeants de la Production française semblent penser que le cinéma est une activité de centre urbain, qu'il commande une certaine masse critique de cinéastes et des infrastructures créatrices et techniques importantes. En région, la densité fait défaut et le mieux à faire, selon eux, dans le meilleur intérêt de la qualité des films, est d'inviter les cinéastes à s'y déplacer. Pas question, dans les circonstances, de régionaliser la production. On voit bien que les deux discours s'opposent, le premier défendant la nécessité d'une expression et d'une culture régionales, le second se faisant l'avocat de normes nationales qui se conjuguent sous le mode centralisateur. Cette opposition n'est pas spécifique aux francophones, car les anglophones des autres provinces canadiennes formulent aussi des griefs analogues contre le siège social.

\section{LA RÉGIONALISATION DES ACTIVITÉS DE PRODUCTION}

C'est d'ailleurs chez les anglophones que s'affirme en premier la nécessité de régionaliser la production. Le 3 mai 1971, Rex Tasker, alors produc-

historique : Jean-Yves Bégin, Le film et les médias communautaires comme instrument d'intervention sociale (1977). Voir aussi Office national du film, Politique culturelle et plan quinquennal — projet (1969) ainsi que Gary Evans, In the National Interest. A Chronicle of the National Film Board of Canada from 1949 to 1989 (Toronto, University of Toronto Press, 1989).

15. Les Acadiens de la dispersion (1967), La Noce est pas finie (1971), Un soleil pas comme ailleurs (1972). Voir Josette Déléas, Léonard Forest ou le regard pionnier (Moncton, Centre d'études acadiennes, 1998). 
teur du studio B de la Production anglaise ${ }^{16}$, envoie au commissaire adjoint André Lamy une note, intitulée Why Decentralize? pour le convaincre de la nécessité de régionaliser la production, ce qui correspondrait - et c'est là une dimension fondamentale - à un changement qu'il perçoit dans les structures politiques, économiques et sociales du Canada. Selon Tasker, le moment serait venu de mettre fin à l'hégémonie du quartier général montréalais et de développer les ressources créatrices dans chaque région, ce qui contribuerait à un nécessaire sens d'identité régionale. L'ONF ne semble pas pressé de bouger dans cette direction, mais il y sera contraint par le gouvernement. Le vent souffle en faveur de la décentralisation au Canada. En effet, au printemps de 1972, à la suite d'une longue période de consultation et de réflexion, le gouvernement décide d'établir une politique nationale du film qui propose notamment le partage avec l'entreprise privée de la production des films commandités et la régionalisation des activités au sein de studios spécifiques. La politique est annoncée par le Secrétaire d'État Gérard Pelletier devant quelque 150 dirigeants de l'industrie cinématographique canadienne. Celui-ci déclare notamment qu'il vise l'ouverture de l'ONF : «J'emploie ici le mot “ouverture” parce qu'il me semble le mieux décrire l'état d'esprit qui régnera désormais à $1^{\prime} O N F^{17}$.» Dans cette foulée, il annonce la création de quatre centres régionaux, sans préciser leur appartenance linguistique. C'est effectivement en 1973 qu'est ouvert le Centre de production de l'Atlantique et Tasker en devient le premier directeur. Or les francophones hors Québec ne sont pas très heureux de devoir s'intégrer dans une production régionale bilingue où ils seront immanquablement subordonnés aux anglophones, alors qu'ils ne cessent de proclamer leur spécificité. De plus - ce qui est presque perçu par eux comme une injure -, l'ONF inaugure en 1973 le Programme de régionalisation du Québec. À leurs yeux, on choisit d'aider les régions du Québec, alors que le reste du Canada français est oublié.

Mais il faut rappeler ici un autre élément contextuel qui démontre qu'il est plus facile d'affirmer "qu'un nouvel état d'esprit régnera» que de le

16. Les Production anglaise et Production française sont subdivisées en studios qui ont à leur tête un producteur. Chaque studio possède une orientation programmatique particulière et cette structure a toujours évolué au fil des ans. Le Studio B est un de ces studios, célèbre par ses innovations esthétiques et de contenu.

17. Notes d'une allocution prononcée à Montréal le 4 juillet 1972 sur la première phase d'une politique $d u$ film, 5. Ce texte se trouve au service des Archives de l'ONF à Montréal, sous la rubrique Film Policy. Dans le même dossier et sur le même sujet, on peut lire le compte rendu de la réunion du Conseil privé du 3 mai 1972, où il est écrit : «The Film Board's Montreal production facilities should be decentralized in favour of regional production units. » 
faire régner. L'atmosphère interne n'est pas au beau fixe à la Production française en 1973. Les cinéastes francophones demandent que l'ONF cesse d'être un instrument de propagande fédérale. Il faut dire que, depuis quelque temps, les interventions du commissaire Sidney Newman à l'égard de certains d'entre eux ne contribuent pas à alléger l'atmosphère. On lui doit notamment la censure d'On est au coton (Denys Arcand, 1970) et de 24 heures ou plus... (Gilles Groulx, 1971), deux films où s'expriment une vision marxiste et révolutionnaire de la société avec laquelle les dirigeants de l'ONF ne sont pas d'accord. Il n'hésite pas non plus à modérer les ardeurs revendicatrices de ceux qui travaillent au programme Challenge for Change/Société nouvelle ${ }^{18}$. C'est en février 1974 que circule un rapport interne où on peut lire notamment qu' "en accord avec la politique de décentralisation et de démocratisation de la culture mise de l'avant par le Secrétariat d'État, la régionalisation d'une partie des activités de la production française est encore nécessaire pour arrêter les effets néfastes de l'impérialisme culturel centralisateur de la ville de Montréal ${ }^{19}$ ". Ce texte explique la filiation avec la politique du Secrétariat d'État — causalité efficiente externe - mais commente également de manière forte et spécifique le fonctionnement de l'Office. Par voie de conséquence, le rapport établit des principes qu'il veut voir appliquer : respecter l'identité culturelle des populations; se servir le plus possible des ressources humaines et financières de leur milieu; mobilité d'action, souplesse d'opération des équipes. Sur le plan de la formation, l'objectif est de repérer et de former des créateurs et des techniciens locaux. Ce sera d'ailleurs la première tâche des responsables régionaux qui verront à organiser des stages et ateliers en région ou au quartier général.

Lorsqu'en 1974, pour se conformer à la politique gouvernementale et aux attentes des francophones, la Production française décide de créer trois centres de production hors du Québec (à Moncton, Toronto et Winnipeg), plusieurs cinéastes québécois de l'ONF et même de l'extérieur vont interpréter ce geste comme une manœuvre fédéraliste destinée à affaiblir le cinéma québécois et à nier sa spécificité en le noyant parmi les autres cinémas francophones canadiens. Ils ne peuvent reconnaître que l'ONF est une institution qui évolue en fonction des politiques définies par l'État (sans nier qu'elles puissent être contradictoires) et non pas exclusivement selon

18. Voir l’interview de Jean-Pierre Tadros, «Sydney Newman à l’ONF. Remettre un peu d'ordre dans la maison ", Le Devoir, 17 juillet 1971.

19. Non signé, Production régionale francophone, 2. 
des priorités qu'elle déterminerait elle-même. La suite des rapports entre le siège social et les centres régionaux, particulièrement celui de l'Acadie, sera surdéterminée par les circonstances qui ont présidé à la création de ceux-ci. Mais il faut préciser que les régions ne définissent pas alors, et ne définiront jamais, leur priorité en fonction du Québec, mais selon leurs besoins propres, alors qu'il faut reconnaître que la Production française s'était surtout donnée comme mission (évidemment sans que cela soit officiel) d'interpréter quasi exclusivement la situation québécoise et de négliger le reste du Canada français. Pas étonnant donc qu'une opposition interne se manifeste contre la création de centres de production locaux, même modestes.

La tension se cristallise autour de la nature des productions : artisanale, semi-professionnelle ou professionnelle ${ }^{20}$. Les activités artisanales demeurent sous la responsabilité des régions, alors que les activités semi-professionnelles passent sous celle des régions mais sujettes à l'approbation de la Production française. Les activités professionnelles s'intègrent par contre à la vie normale de la Production française et les projets sont soumis au Comité du programme ${ }^{21}$. Pour assurer le contenu et le succès de son programme de régionalisation, l'ONF choisit de créer dans chaque région une structure originale qui doit aider le producteur délégué à déterminer la pertinence des documents à produire et favoriser leur diffusion. Il s'agit du Comité d'action régionale (CAR), une structure qui s'inspire des comités de citoyens, qui fleurissent alors au pays, et qui participe de la même idéologie. L'esprit local est très affirmé : on veut des créateurs locaux bien ancrés dans la réalité régionale et accessibles à leur milieu. On veut que le centre appartienne à la population. On veut enfin se servir des médias comme moyen d'action culturelle, sociale, politique et économique ${ }^{22}$. Cette concep-

20. Instaurer une telle hiérarchie au sein d'un même organisme est assez inusité. Évidemment ce qui est qualifié d'artisanal vise des productions bon marché, réalisées par des cinéastes de peu d'expérience, sinon néophytes, et bénéficiant de moyens techniques réduits. Leur marché est local et communautaire. Tout à l'opposé de la production onéfienne provenant du siège social, par définition professionnelle.

21. Structure décisionnelle créée en 1968 dans une perspective autogestionnaire pour assurer le contrôle des cinéastes sur la production et ne pas le laisser uniquement à la direction. Ayant à sa tête un directeur élu parmi ses pairs, le Comité regroupe des employés de la production et de la distribution et sa composition varie continuellement. Son directeur se voit doté à l'origine d'un pouvoir presque aussi grand que celui du directeur de la Production qui est membre d'office de cette instance qui reçoit et étudie les projets de films, puis décide de leur mise en œuvre (que ce soit à l'étape de la recherche, de la scénarisation ou de la réalisation). Précisons tout de suite que le Comité est presque exclusivement composé de Québécois.

22. Procès-verbal de la réunion tenue à Bathurst les 9 et 10 juillet 1974, 5 p. 
tion militante de l'audiovisuel, très en prise sur l'époque, rappelle plus la tendance "Société nouvelle» que celle du programme régulier de l'ONF.

L'ONF effectue dès l'automne de 1974 un premier bilan de son action. S’il accepte de déléguer aux régions le pouvoir de concevoir leurs projets et d'identifier leurs besoins librement, en tenant compte des lignes générales correspondant au mandat et aux limites de l'ONF, il maintient que doivent être centralisés au Comité du Programme français les projets d'envergure nationale qui s'adresseraient à un auditoire qui n'est pas préoccupé des problèmes spécifiques à une région donnée ${ }^{23}$. Un an plus tard, rencontrant la presse, le producteur responsable des régions, Jean-Marc Garand, notera que chaque région possède déjà sa personnalité : socioculturelle dans les Maritimes, culturelle dans l'Ouest et à part égale politique et culturelle en Ontario $^{24}$. Le rapport annuel met d'ailleurs l'accent sur le même fait quand il écrit : «Le producteur délégué a entrepris, en collaboration avec un Comité d'action régionale, un programme de repérage de cinéastes et d'artisans, d'entraînement et de production artisanale. On peut voir dans cette ouverture de l'ONF la promesse à long terme d'une interprétation du pays encore plus authentique puisque réalisée par des cinéastes vraiment enracinés dans le milieu qu'ils filmeront ${ }^{25}$.»

L’ONF établit la nécessité de tenir une réunion annuelle des représentants régionaux de la production, de la distribution et des CAR dans le but de faire le point mais surtout d'orienter les attitudes de la Production française ${ }^{26}$. Lors de la première rencontre, les trois producteurs des régions exposent leur philosophie et leur action. Le procès-verbal tire la conclusion suivante : «Au cours de cette rencontre, il devient évident que nos activités de régionalisation ont été assumées par des producteurs délégués dont le discours est à la fois lucide, vigoureux et cohérent. Si leur langage trahit parfois une idéologie autonomiste assez surprenante pour la métropole, on s'est demandé qui pourrait bien leur jeter la première pierre ${ }^{27}$."

23. Voir Jean-Marc Garand, État et prospective (Studio D, novembre 1974), 13-14; J.-M. Garand, Régionalisation de la production française (novembre 1974, 3 p.); Régionalisation de la Production française (novembre 1974, 3 p.). À noter que l'ONF envisage aussi au même moment de mettre sur pied six centres de production au Québec.

24. Murray Maltais, «La régionalisation. À l’ONF, le cinéma se démocratise », Le Droit, 13 septembre 1975 ; Claude Daigneault, "À qui profite l'ONF? La production française », Le Soleil, 27 septembre 1975 .

25. Office national du film, Rapport annuel 1974-1975, 11.

26. Bilan et prospectives du programme de régionalisation de la production française (Compte rendu de la réunion des 8-10 décembre 1974).

27. Comité du programme français, Procès verbal de la $259^{e}$ réunion (6 février 1975). 


\section{LES PROBLÈMES FINANCIERS MENACENT LA RÉGIONALISATION}

Les pierres viendront bien assez tôt... En fait moins d'un an plus tard. La situation financière sert d'événement déclencheur. Les producteurs régionaux trouvent qu'ils n'ont pas suffisamment d'argent pour réaliser leurs projets et payer décemment ceux qui y travaillent. L'écart entre les professionnels de Montréal, payés selon le principe d'achat de services au quartier général, et les acteurs locaux est d'ailleurs clairement souligné. Le gouvernement fédéral vient en outre de leur retirer plusieurs sources de financement dont, parmi les plus importantes, les programmes Perspective-Jeunesse et Projets d'initiative locale. Par contre, en février 1976, à Montréal, les quatre cinéastes membres du Comité du programme démissionnent pour marquer leur désaccord avec la direction de la Production française. Ils trouvent que le budget régulier (dit "vote culturel») a tendance à stagner, tandis que les programmes spécifiques (c'est-à-dire indépendants du budget de la Production française) jouissent à leurs yeux d'une prépondérance croissante; ils égaleraient le précédent. Il faut savoir que le programme «Société nouvelle» a été reconduit en 1975 pour cinq ans et que la Régionalisation hors Québec va chercher $340000 \$$ en argent externe ${ }^{28}$. Si on exprime cela en termes plus crus, cela veut dire qu'on veut bien proférer de beaux discours idéologiques qui affirment la primauté de la base comme lieu d'origine du dynamisme et de l'esprit de la population, dans la mesure où cela signifie que le centre soit la source de cette expression, que la régionalisation n'entraîne pas des coûts d'exploitation accrus pour le siège social et que la formation d'une nouvelle génération de cinéastes dans les régions ne puisse signifier une éventuelle concurrence. L'idée des «voleurs de job» n'est pas loin. Si on ajoute à cela le fait que la direction de la Production française aimerait que les réalisateurs montréalais augmentent un peu leur rendement — ce à quoi ces derniers s'opposent au nom de la liberté de la création et des exigences intrinsèques des œuvres - on comprend bien que tous les ingrédients sont en place pour un heurt régions-Montréal.

28. Bien qu'elle ait évolué au fil des années, la question des budgets au milieu des années 1970 peut se résumer ainsi. Le parlement vote un budget annuel (27 714000 \$ en 1977) auquel s'ajoutent les revenus provenant des ministères (commandites) ou pour des programmes spéciaux comme Challenge for Change/Société nouvelle ainsi que les recettes de la distribution (ajout total : $9842000 \$$ en 1977). Ces sommes sont réparties entre les différents services : Production (55\%), Distribution (35\%), Administration (10\%) et en immobilisation. Les Productions française et anglaise reçoivent chacune un montant global qu'elles doivent répartir entre tous les studios pour leur programme général (c'est-à-dire les films dont elles décident de la réalisation) au nombre desquels on retrouve la Régionalisation -, pour les commandites et autre programme spécial, pour les versions et adaptations et pour les productions multimédias. 
Les membres des CAR sont conscients de la crise financière qui frappe l'ONF et de la vive opposition de l'Atelier des cinéastes ${ }^{29}$ au programme de régionalisation. Ils n'ont guère apprécié que, pendant la crise de septembre 1975 découlant du gel des prix et des salaires annoncé par le gouvernement Trudeau, ils aient été obligés de subir des coupures en raison des difficultés de la Production française. Ils estiment que celle-ci n’a pas de droits sur l'argent des régions. Ils en débattent franchement avec des représentants de Montréal qui leur rendent visite en mars 1976. Ils apprennent à cette occasion que le Comité du programme souhaite les rencontrer pour évaluer leur travail. Cette rencontre, qui a lieu en juin, "pose un problème aux vieux routiers de l'Office qui, tout en se défendant de paternaliser, ne peuvent s'empêcher d'exposer les exigences d'apprentissage des différents métiers du cinéma qu'ils ont appris à maîtriser ${ }^{30}$ ». Les membres du Comité doutent du fait que les régions puissent assurer la continuité, car il y manque de compétences. Le directeur du Comité, le réalisateur Clément Perron, y va alors d’un commentaire acéré : «Née presque malgré nous, la Régionalisation hors Québec promène déjà dans les couloirs de l'Office son dossier noir d'exilée, que l'asphyxie guette, mais elle promène aussi un visage imberbe d'adolescent frustré qui arrive mal à dissimuler, sous ses gestes brusques et ses verres teintés, le désarroi de son âge, qu'un grand espoir inavoué habite, on le sait bien ${ }^{31}$.»

Ce texte traduit assez bien le sentiment de Montréal et le directeur de la Production française, Yves Leduc, y fait écho de la manière suivante : «Estce que ça vaut la peine de faire la régionalisation hors Québec si la [production] francophone se meurt ${ }^{32}$ ? " À ce questionnement les producteurs régionaux répondent qu'il leur est nécessaire de prendre conscience de leur identité canadienne-française. Ce bref compte rendu des débats, du type dialogue de sourds, expose bien le fossé qui sépare Montréal des régions et indique qu'en plus du contexte du premier plan quinquennal qui entraîne des mesures d'austérité, la question nationale québécoise détermine l'attitude des cinéastes du quartier général envers les régions. Les producteurs

29. Alors que depuis 1968 existe à l'ONF le Syndicat général du cinéma et de la télévision qui regroupe en une seule unité de négociation les membres de tous les métiers œuvrant pour l'organisme, les cinéastes francophones qui occupent des fonctions plus créatrices vont aussi se regrouper au sein d'un Atelier pour défendre les conditions d'exercice de leur métier et non pas leurs conditions de travail.

30. Comité du programme français, Procès-verbal de la $302^{e}$ réunion (10 juin 1976), 3.

31. Ibid., 4.

32. Régionalisation. Procès-verbal de la réunion d'évaluation tenue à l'ONF du 7 au 11 juin 1976, 18. 
des régions ne sortent pas rassurés de la semaine d'évaluation, mais ils ne modifient pas de manière significative leur fonctionnement. Tout juste ontils mesuré les griefs du siège social. Le réalisateur et producteur Guy L. Coté les rappellera quelques années plus tard ${ }^{33}$. Selon lui, il ne faut pas abdiquer la préoccupation nationale au profit d'un séparatisme culturel régional, qu'il soit albertain ou acadien, bien que l'ONF soit un microcosme de la société canadienne, les pôles national/régional connaissant les mêmes tensions que le pays dans son ensemble. Le programme de régionalisation de l'Office n'ayant pas reçu de la part de l'État fédéral le financement nécessaire à sa continuation, l'administration a dû couper dans la mission dite «nationale» afin de poursuivre la décentralisation. L'effet sur les cinéastes du quartier général fut catastrophique car leur existence était menacée. Or comme le dira l'un d'eux, Jacques Godbout, «s'il n'y a pas de centre, il ne peut y avoir de régions ${ }^{34}$ ».

Ce texte, où Montréal se drape curieusement derrière le mot «national», en autant que ce vocable désigne le centre, laisse clairement entendre les griefs et les discours de légitimation du siège social (comprendre des cinéastes québécois) à l'encontre des régions. Pourtant celles-ci ne roulent pas sur l'or. Or il ne faut pas oublier que la régionalisation est une politique ministérielle contre laquelle tous les atermoiements des cinéastes de Montréal ne peuvent rien. Mais ceux-ci n’entendent pas cette logique politique et font comme si l'ONF n'obéissait qu'à une logique de cinéma. En mars 1977, comparaissant devant le comité des Communes chargé d'examiner les crédits de l'ONF, le commissaire André Lamy rappelle d'ailleurs aux députés que la régionalisation est une priorité. C'est pourquoi le budget total de la régionalisation, qui était à l’origine de 900000 , atteint 2950000 \$ en 1977 et pourrait atteindre $7900000 \$$ en 1981. Concrètement, en 1977, la production française hors Québec reçoit 15\% du budget total de la Production française.

Le cinéma aide les habitants des régions à prendre conscience de la vie des collectivités qui les composent et de leur réalité sociale, économique et culturelle. S'ils sont tournés en région, la majorité de ces films sont montés et terminés à Montréal où se retrouvent les équipements techniques nécessaires. On ne peut pas dire que les cinéastes hors Québec soient les

33. Chiffres en main. 25 ans de cinéma à l'Office national du film (février 1985), 24-28. Il s'agit là d'une réflexion critique sur le projet de plan quinquennal d'exploitation présenté par la direction de l'ONF en octobre 1984.

34. Cité par Coté, 28. 
bienvenus dans les corridors du siège social. L'administration montréalaise tentera de contourner le problème avec des tactiques comptables qui, incidemment, vont hérisser les régions. C'est le cas avec Kouchibouguac (réalisation collective, 1978) et Le Manitoba ne répond plus (Raymond Gauthier, 1977), où on décide pour la première fois d'affecter des fonds de la Régionalisation hors Québec à des activités de production professionnelle $\mathrm{e}^{35}$, ce que Montréal présente comme une progression vers le cinéma professionnel, mais que les régions considèrent comme un détournement de fonds. Les cinéastes de Montréal n'apprécient guère les régions en ce début de 1977, particulièrement parce qu'ils se méfient de structures de programmation qui échappent au Comité du programme ou au syndicat. C'est d'ailleurs lors d'une assemblée syndicale en mars 1977 que Jacques Godbout déclarera : «L'ONF se retrouve hélas avec des classes maternelles. [...] Le cinéma à Winnipeg ou Moncton ne sera toujours qu'amateur avec des outils professionnels. [...] La régionalisation n'encourage pas, maintenant que nous en avons fait l'expérience, la qualité. Le label ONF s'y déva$\operatorname{lue}^{36}$.» Quelqu'un d'autre ajoutera même à cette occasion : «Il y a trop de talents au Québec qui crèvent de faim pour donner de l'argent aux Acadiens, aux Franco-Ontariens ou aux Franco-Manitobains ${ }^{37}$.»

On comprend bien qu'à l'époque, la situation ne s'améliore pas entre les deux pôles, plus spécifiquement au niveau du Comité du programme. Les producteurs et les cinéastes hors Québec ne veulent pas être soumis à ce Comité et refusent d'être ses otages. On sent qu'une partie de bras de fer se joue entre les parties. On le devine, tout tourne autour de la question de la qualité des films et du professionnalisme. Pour résoudre le problème, le Quartier général décide de créer un poste de producteur qui aura la responsabilité des régions et qui réunira chaque mois les trois producteurs délégués ${ }^{38}$, et de mettre sur pied une commission itinérante qui évaluera les opérations et l'orientation du programme de régionalisation hors Québec. Signe des doutes qui hantent les esprits montréalais, Garand s'interroge même, lors du Comité du programme du 16 juin, sur la pertinence de la Régionalisation. De telles décisions hérissent les régions qui

35. Ces activités désignent les réalisations des cinéastes relevant du siège social. Théoriquement les fonds des studios régionaux ne peuvent leur être affectés.

36. Cité dans Georges-André Prud'homme, Les p'tites vues des héritiers de lord Durham (Toronto, 12 juillet 1977), 2.

37. Ibid., 2.

38. Ce sera Marc Daigle qui le 8 novembre assumera cette responsabilité de coordination technique. 
continuent à revendiquer. Des rumeurs se mettent à circuler. Le commissaire André Lamy rencontre les producteurs régionaux pour obtenir plus d'informations. Croyant à une revendication d'autonomie régionale, il annonce d'emblée qu'il refuse la balkanisation de l'ONF. Mais les producteurs répondent que leur demande vise seulement la création d'une entité régionale autonome (à la rigueur française et anglaise) qui relèverait directement du commissaire adjoint ${ }^{39}$. Le projet restera lettre morte dans l'immédiat, non sans avoir donné lieu au passage à des échanges virulents. Il faudra que le commissaire adjoint, James de B. Domville, intervienne pour arbitrer les débats. Mais l'ONF ne changera jamais de point de vue : la régionalisation relève de la Production et les films professionnels relèvent d'elle, car l'expertise réside à Montréal. Pour calmer toutefois le jeu, la Production française propose des mesures qui lui semblent faire preuve d'ouverture. Le CAR-Acadie ne se laisse pas amadouer. Il réplique en demandant d'avoir de droit un représentant au Comité du programme. Évidemment, la Production française refuse. Le CAR-Acadie rétorque en affirmant : «Si la régionalisation doit exister, la programmation en région est une condition essentielle. Ou bien, nous sommes des citoyens à part entière, ou bien nous ne le sommes pas. Sur ce point, il n'y a plus rien à dire; tout a déjà été dit. Nous voulons être chez nous, chez nous, autant dans le cinéma qu'ailleurs ${ }^{40}$.»

\section{TENSIONS CENTRE/RÉGIONS : MENACES DE FERMETURE}

Tel est l'état d'esprit qui règne en 1978. Cet été-là, l'ONF est frappé par des restrictions budgétaires. Une coupe de $4000000 \$$ de moins pour tout l'ONF, $500000 \$$ pour la Production française, environ $25 \%$ de son budget. Celleci refuse de couper parmi les permanents, le «cœur de son être». Elle arrête quelques mesures : réduction radicale des activités et des coûts fixes, diminution du personnel pigiste. Mais la mesure qui vient en tête de liste, c'est la disparition du programme de régionalisation, une solution que la direction de la Production française définit de la sorte : il s'agit d'un «choix entre le cour et le doigt malade ${ }^{41}$ ». En même temps, on se rend compte que cette position a des conséquences politiques. Évidemment. Les trois producteurs régionaux ne tardent pas à réagir. Ils trouvent inacceptables les

39. Georges-André Prud'homme, Note de service à Paul-Eugène Leblanc et René Piché (Toronto, 4 juillet 1977).

40. Comité d'action régional — Régionalisation Acadie, Un exemple (non daté), 3.

41. Jean-Marc Garand, Résumé, présentation et explication du document «Modalités d'application des restrictions budgétaires» (18 septembre 1978), 1. 
principes et les raisons invoqués par le centre et rappellent le mandat national de l'ONF. Il est clair que, pour eux, les cinéastes de Montréal essaient de s'en tirer sur leur dos. Au mieux accepteraient-ils une réduction proportionnelle. Ils font pression sur Lamy pour qu'il intervienne auprès du Conseil d'administration ${ }^{42}$. Ils vont même le faire directement eux-mêmes tant ils se méfient du commissaire. La partie se corse, car le commissaire entérine la recommandation de la Production française et s'apprête à la soumettre à la réunion du Conseil d'administration de septembre.

Les régions n'ont plus d'autre choix que de réagir. Mais le pire arrive presque. Le CA décide de fermer, le 31 décembre 1979, deux régions mais refuse de toucher à l'Acadie pour des raisons culturelles et à cause du nombre de sa population francophone. Le commissaire s'attire de la part d'un journaliste acadien l'observation suivante : «Finalement, d'une façon ou d'une autre, vous faites, vous vous trouvez à faire une espèce de sélection naturelle en disant qu'hors du Québec et de l'Acadie, il n'y a point de salut pour les francophones comme certains veulent le dire ${ }^{43}$.» Comme il fallait s'y attendre, les régions n'embarquent pas dans ce «diviser pour régner» et réagissent en front commun. Les CAR, la rage au cœur, crient à l'injustice, à l'arbitraire et à la discrimination. Ils refusent à l'ONF le droit de décider de la fin des francophones et dénoncent, en se comparant à la régionalisation anglaise, les «deux poids, deux mesures » qui s'appliquent ${ }^{44}$. Les débats de la Chambre des Communes font écho à toute cette affaire. Mais le secrétaire d’État John Roberts décide de ne pas renverser la décision du conseil d'administration de l'ONF. Il se ridiculise même, à notre avis, en proposant aux régions de former leurs cinéastes en puisant dans les programmes de soutien aux minorités linguistiques ${ }^{45}$.

Les CAR ne se donnent pas pour vaincus. Ils continuent leur lobby en réaffirmant les principes fondamentaux qui guident leur action, à savoir que la régionalisation française soit reconnue comme une composante essentielle de l'ONF, qu'elle devienne une unité de production relevant directement du Commissaire et que la programmation en région relève directement du producteur en région et du $\mathrm{CAR}^{46}$. Ceux-ci s'occupent

42. Paul-Eugène LeBlanc, René Piché, Georges-André Prud'homme, Lettre à André Lamy (20 septembre 1978), 3. Les mêmes, Lettre au Conseil d'administration (21 septembre 1978), 4.

43. Entrevue avec André Lamy (Radio-Canada - Moncton, 26 septembre 1978).

44. Office national du film. Régionalisation/Acadie, Communiqué de presse (23 septembre 1978), 3.

45. «Roberts refuse de renverser la décision de l’ONF», Le Droit, 22 décembre 1978.

46. Communiqué de presse des Comités d'action régionale Ontario, Ouest et Acadie, Coupures de la régionalisation française de l'Office national du film en Ontario et dans l'Ouest (19 février 1979). 
aussi de mobiliser la population, de susciter des appuis et de faire pression sur les politiciens. Pendant qu'ils s'activent, la situation évolue. Excédés par les agissements du siège social, les producteurs délégués de l'Ontario (Georges-André Prud'homme) et de l'Acadie (Eugène LeBlanc) remettent leur démission qui doit entrer en vigueur un an plus tard, au début de 1980. Le gouvernement, qui entre en période électorale, n'aime guère ce genre de controverse. Il demande au sous-secrétaire d'État Pierre Juneau de prendre en main le dossier ${ }^{47}$. Mais celui-ci perd son poste à la suite de l'élection de mai 1979 qui porte le gouvernement conservateur minoritaire de Joe Clark au pouvoir. Ce même mois, Jean-Marc Garand devient directeur de la Production française. Il jongle avec l'idée de rendre les programmes de régionalisation partie intégrante de l'activité de l'ONF. Plusieurs se rappellent les coupes budgétaires du gouvernement libéral et se méfient du nouveau gouvernement conservateur. Les régions accentuent donc la pression, ce qui finit par porter fruit, car lors de sa réunion de décembre ${ }^{48}$, le Conseil d'administration de l'ONF annonce sa décision d'assurer la continuité des activités des régions francophones hors Québec. En apparence, le problème est réglé.

Mais l'ONF entre dans une décennie où ses moyens de production commencent à décliner alors qu'il est impossible de réduire les dépenses en région. Au contraire. Les directives sont à l'effet d'étendre les activités sur l'ensemble du territoire canadien pour répondre à l'objectif politique de refléter le paysage socioculturel et français du pays. Les CAR et les producteurs régionaux ne s'en tiennent pas là. Ils reprennent le collier. Sûrs d'avoir rempli leur mandat original de formation et de production, ils en énoncent un nouveau en paraphrasant le texte de la loi qui régit l'ONF : «Interpréter, par le cinéma, les réalités de nos régions respectives à nos populations d'abord et à l'ensemble de la population canadienne par la suite $^{49}$.» Leur objectif est maintenant de garantir la permanence, la stabilité

47. Administrateur à l'ONF depuis 1954 et premier directeur de la Production française en 1964, il connaît bien la maison et plusieurs des intervenants au dossier. Cet ami de Pierre E. Trudeau quitte l'ONF en 1966 pour passer au CRTC. En 1975, il est nommé ministre des Communications, mais il démissionnera faute de parvenir à se faire élire au Parlement. Il occupera dès lors plusieurs postes de haut fonctionnaire avant de devenir, en 1982, président de la Société Radio-Canada.

48. Régionalisation française hors-Québec, Présentation au Conseil d'administration de l'Office national du film (14 décembre 1979), 6. Ce même mois, le gouvernement Clark est renversé aux Communes, ce qui pave le retour de Pierre E. Trudeau en février 1980.

49. Programme Régionalisation hors Québec, Régionalisation. Planification 1979-1980 (mai 1979), 3. 
et le développement de la régionalisation. Ils reviennent à la charge avec l'idée d'un secteur "régionalisation" autonome, seule condition pour que celle-ci soit autre chose qu'une décentralisation, et de la transformation en postes réguliers des postes de producteurs délégués. Réuni en mars 1980, le CA entend une partie de ces arguments sans acquiescer tout à fait à l'autonomie de la Régionalisation hors Québec. La direction de l'ONF réagit diplomatiquement en créant en mai un studio spécifique pour la régionalisation, le Studio E, avec un chef de studio, Raymond H. Gauthier, qui provient lui-même de l'extérieur du Québec. Son mandat est de planifier et de coordonner avec les producteurs régionaux le programme de production, de tenir la direction au courant de tous les aspects de la production audiovisuelle et de participer à l'élaboration des objectifs, des principes directeurs et des programmes de la Production française, suivant les directives qui lui sont transmises par les producteurs régionaux. Les films régionaux ont donc le même statut que les autres films ${ }^{50}$.

Ce renforcement de la région ne fait pas l'affaire de tout le monde. Venant à peine d'être réélu à la tête du Comité du programme, Clément Perron monte à nouveau aux barricades avec un pamphlet qui a le mérite de dire tout haut le sentiment de la majorité des cinéastes montréalais ${ }^{51}$. Il n'y va pas de main morte. Un florilège de citations va nous donner la saveur du texte :

Ce n'est pas parce qu'une chose a été partie tout croche et sur des bases inappropriées qu'il faut accepter qu'elle soit prolongée telle quelle.

L'ONF n'est pas un C.L.S.C. ni une maison de services à la disposition du tout venant. L'ONF est une maison de production et de distribution de films basée sur une organisation industrielle et artistique intégrée. [...] Cette expertise professionnelle, toujours en mouvance, constitue maintenant une sorte de cahier des charges et garantit à la fois la pertinence actuelle et l’avenir de la Production française.

Le fond de ce problème concerne l'apprentissage et la formation professionnelle impossible à s'auto-réussir [sic] dans des milieux sans maîtres ni tradition, des milieux sans «milieu» où les bassins culturels sont par ailleurs impuissants à générer et à supporter toutes les structures et les infrastructures nécessaires au discours cinématographique. 
Si nous envisageons une démarche sérieuse vers le professionnalisme, $[\ldots]$ le CAR, tel qu'on le connaît, n'a aucun rôle à jouer. Il est plutôt un instrument lourd, coûteux et inefficace.

Et le bouillant cinéaste continue sur la même lancée : l'ONF démissionne de son rôle, abdique ses responsabilités, commet un crime de lèsecinéma en laissant la production en région aux mains de producteurs sans métier et de CAR improvisateurs. L'ONF ne doit pas accepter l'autoprogrammation d'amateurs qui mettent en production des brouillons. Il conclut de manière spectaculaire et provocatrice : "Il faudrait décorer d'une anti-médaille celui qui a laissé croire à la nécessité d’une production cinématographique dans/par/pour toutes les minorités francophones du pays. »

Entre celles-ci et Montréal, le torchon brûle à nouveau. Le débat reprend, les réunions aussi. Dès l'été de 1980, on se remet à se rencontrer pour à nouveau préciser le mandat de la régionalisation et expliciter les règles en ce qui concerne la programmation. On veut aller en profondeur dans cette question. Mais les intervenants ne se rejoignent pas. Aux premiers qui constatent des lacunes graves côté encadrement professionnel et qui disent vouloir s'en tenir à parler cinéma, les seconds rétorquent qu'ils passent le plus clair de leur temps en batailles politiques et que les questions politiques déterminent la production. Face aux Montréalais qui trouvent que Perron pose les bonnes questions, les représentants hors Québec parlent d'insultes et d'attitude montréalaise fermée. Mais de fil en aiguille, un certain consensus se dégage autour de trois idées : refléter et interpréter les préoccupations des collectivités régionales vis-à-vis d'ellesmêmes et vis-à-vis des autres; produire un cinéma de qualité ; favoriser une promotion intensive et qualitative dans les différentes communautés. On constate qu'en accord avec le mandat de l'ONF, la régionalisation doit favoriser une participation des francophones hors Québec à la cinématographie nationale ${ }^{52}$.

\section{NOUVEAUX HEURTS AUTOUR DE LA PROGRAMMATION. UNE FRONDE SE DESSINE}

Ce consensus va quand même buter sur sa mise en application. Il appert en effet assez vite que les parties n'ont pas la même vision d'avenir pour la régionalisation ni la même vision quant à son intégration au processus 
de programmation. La régionalisation doit-elle se définir uniquement en fonction de l'ONF et de la Production française, ou peut-elle bénéficier d'une sorte de clause dérogatoire? La question est posée. Parce qu'elle encourage le seul agent cinématographique français hors Québec, la régionalisation est une nécessité à laquelle la Production française ne peut échapper. Toutefois, avec l'inflation, les coûts de production augmentent et le programme hors Québec gruge quelque $20 \%$ de son allocation budgétaire, ce qui soulève plusieurs questions au sein de la direction de la Production. Pour «rentabiliser» ces productions, on souhaite que les films produits, tout en demeurant centrés sur les réalités des francophones hors Québec, traitent leur sujet de façon plus universelle et renforcent par le fait même le mandat national du programme. Par ailleurs les régions n'échappent pas aux nécessités de la coproduction, tout comme le programme général qui doit de plus en plus coproduire avec Radio-Canada ou avec le privé, ce qui est d'ailleurs une politique ministérielle. Dans les régions, il existe peu, sinon pas du tout, d'entreprises privées. Il faut en faire apparaître. Ainsi, que ce soit en Acadie, en Ontario ou dans l'Ouest, on verra naître au fil des ans de petites compagnies locales.

Si l'Ontario et l'Ouest acceptent sans enthousiasme ces règles du jeu, l'Acadie résiste. Elle n'aime pas le mode de fonctionnement envisagé et se méfie d'idées comme «la recherche de thèmes plus universels tout en étant acadiens ». Elle accuse Montréal de vouloir faire indirectement main basse sur le budget de la régionalisation et de laisser des broutilles aux Acadiens, d'imposer ses priorités de programmation et ses paramètres de production, d'abandonner la vidéo et le type de fonctionnement qu'elle suppose. Selon elle, l'enjeu est clair. Accepter la planification centrale signifie accepter de voir disparaître la régionalisation d'ici deux ans. La résistance s'organise et vient de partout. Clément Perron, qui est alors directeur du Comité du programme, ira d'un commentaire : «Des interlocuteurs valables seront bientôt trouvés aussi là-bas qui comprendront la nécessité de professionnaliser leur démarche plutôt que la bêtifier en la politisant ${ }^{53}$.» La Région rétorque en mars par une planification originale ${ }^{54}$ par laquelle elle dit vouloir assurer son avenir. Point par point elle refuse la planification de Montréal ou la reformule en ses propres termes. Ainsi elle se dit d'accord

53. Commentaire consigné au procès-verbal de la réunion du Comité du programme (28 mai 1981).

54. Régionalisation Acadie, Planification 81-86 (mars 1981). Ce texte de 33 pages est suivi d'un autre le mois suivant qui reprend plusieurs des pièces au dossier : Comité d'action régional, Dossier de la Régionalisation Acadie de l'ONF ( ${ }^{\mathrm{er}}$ avril 1981). 
avec un cinéma professionnel «chez nous, fait par des gens de chez nous en parfaite concordance avec le mandat de l'Office». Budget, financement, formation, rôle du CAR et de la collectivité dans son ensemble, permanence des cinéastes en région, changement au poste du chef du studio $\mathrm{E}$, abolition des trois postes de contrôleurs de qualité au sein du studio E, tout y passe. La Production française ne se laisse pas impressionner et riposte. Garand impose le 18 mars un moratoire au cinéma régional et prépare ses directives. Le moratoire est levé un mois plus tard en même temps que la Production réaffirme ses objectifs, lesquels font l'objet d'un communiqué de presse le 15 avril. Cette fois, le CAR-Acadie s'insurge et rejette unanimement les directives de Montréal. Il ne reconnaît plus Raymond Gauthier, parle de pouvoirs et de droits rapatriés unilatéralement, et décide d'en appeler au commissaire et, si ça ne suffit pas, à la population dans son ensemble. Garand congédie le producteur Acadie, Rhéal Drisdelle, et décide de la fermeture temporaire du bureau de Moncton. La production elle-même est suspendue.

La situation dégénère. La fronde devient politique. La majorité des cinéastes acadiens qui ont travaillé à l'ONF se mobilisent pour sauver leur spécificité. Ils se réunissent chaque semaine et établissent des moyens d'action. Au cœur du problème, on retrouve l'autonomie revendiquée par les cinéastes dans le choix de la programmation régionale et donc le refus d'une quelconque soumission au Comité du programme. Ce que la Production française présentait comme une question cinématographique - le professionnalisme - est devenu maintenant une bataille politique. Pour s'en sortir, la Production française demande en mai à la Société de recherches en sciences du comportement (Sorécom) de faire une étude sur la situation du cinéma d'expression française en Acadie, étude qui se pencherait sur la représentativité du Comité d'action régionale. Cela donne au studio E le temps de souffler et d'essayer de faire en sorte que la vie continue dans les deux autres régions. Sans être aussi radicales que l'Acadie, celles-ci n'approuvent pas nécessairement l'impasse générée par les attitudes des deux parties et auraient préféré des compromis, le temps que l'Acadie réfléchisse et puisse être apprivoisée. Mais ce recours à Sorécom ne fait pas vraiment l'affaire de tout le monde en Acadie. Le CAR réagit en demandant même que le Comité d'étude de la politique culturelle fédérale (ApplebaumHébert) examine en profondeur la structure de l'ONF et ses politiques face à la régionalisation. On veut pousser le débat vers un autre forum, quitte à ouvrir une boîte de Pandore. Pour calmer le jeu, le commissaire adjoint François Macerola se rend à Moncton le 23 juin pour rencontrer les pro- 
testataires. De retour à Montréal, il amende le mandat de Sorécom pour y inclure des éléments qui correspondent mieux aux griefs qu'il a entendus : le professionnalisme, la continuité du travail des cinéastes, les allocations annuelles, les mécanismes de programmation en région, la composition du CAR, les rôles respectifs du CAR et du Comité du programme ainsi que du chef de studio et du producteur délégué. On perçoit que les Acadiens ont tendance à considérer comme leur appartenant les institutions gouvernementales implantées chez eux, surtout les fédérales.

\section{RÉORGANISATION ET REPRISE EN MAIN DES RÉGIONS}

Sorécom remet son rapport à l'ONF en septembre, un rapport de 97 pages! On y met en doute la représentativité du CAR-Acadie et on avance l'idée de trouver une structure plus représentative. En fait il s'agit là d'un des points de l'entente intervenue entre l'ONF et la Société nationale des Acadiens (SNA) qui joue en l'occurrence un rôle de médiateur ${ }^{55}$. Les principes de cette entente sont les suivants : les Acadiens témoignent d'une volonté ferme de réaliser un cinéma authentiquement acadien et l'ONF veut respecter cette volonté et les particularismes de cette même culture; d'autre part, l'ONF a une double responsabilité artistique et administrative en ce qu'il doit gérer ses fonds et répondre de la qualité de son produit. La SNA hérite du mandat de former ce nouveau comité. L'ONF a donc réussi à isoler les protestataires en s'alliant avec la SNA. Sa priorité est alors de dénicher un nouveau producteur qui puisse obtenir l'accord des parties. Le choix se porte sur un Français, Éric Michel ${ }^{56}$. C'est en janvier 1982 qu'a lieu la réouverture du bureau de Moncton. La crise a laissé des séquelles. Éric Michel adopte une attitude ferme et veut repartir sur de nouvelles bases. Il préfère repartir à zéro avec des réalisateurs qui n’ont affronté personne de l'ONF. La Production française/Acadie poursuit sa réorientation tambour battant avec des moyens modestes. Car, entretemps, la situation financière de l'ONF s'étant dégradée, les régions vont encore manquer de moyens financiers. On les encouragera donc à faire des choix économiques et les cinéastes auront peu d'occasions de faire du

55. Par un communiqué de presse conjoint daté du 11 mai 1981, la Société Saint Thomas d'Aquin, la Fédération des Acadiens de la Nouvelle-Écosse, la Société des Acadiens du NouveauBrunswick et la Société nationale des Acadiens annoncent le mandat confié à la SNA. Le rapport Sorécom fait état des discussions entre celle-ci et la direction de l'ONF.

56. Correspondant de l'ORTF au Canada, celui-ci est resté au pays au terme de son mandat et a ouvert à Moncton une maison de services. Il présente le double avantage d'être neutre dans le débat et compétent. 
cinéma. La conclusion du document de planification 1982-1983 est on ne peut plus explicite : "Il est clair que le cinéma régional ne pourra remplir son mandat en 82-83, qu'il devra sauver son cinéma en développant des mécanismes aptes à en assurer la qualité et qu'il devra rationaliser toute dépense d'énergie et d'argent en fonction du cinéma devenu son seul argument d'existence ${ }^{57}$.» En un mot comme en mille, les régions ont des moyens très limités.

Ce qui explique toute cette situation, c'est le dépôt du rapport ApplebaumHébert qui n'est pas tendre pour l'ONF ${ }^{58}$. Le Studio E réagit publiquement au rapport par un communiqué intitulé Le Cinéma régional francophone de l'Office national du film : expression d'une dimension culturelle importante pour le collectif canadien. Le ton est résolument politique, affirmant que seul l'État, par l'entremise de l'ONF, peut permettre aux francophones hors Québec de participer de façon significative et continue au cinéma régional. Il n'empêche que l'ONF doit penser à des réductions budgétaires et à des fermetures. Le bureau de Moncton est épargné car il vient de subir une restructuration en profondeur. Par ailleurs dans un texte de réflexion adressé à tout son personnel, le directeur de la Production française, Jean-Marc Garand, traduit le sentiment d'une bonne partie du personnel à l'égard de ce rapport : «Il est malheureux que ce soit sous la pression environnementale d'un rapport aussi peu réfléchi, spécialement au niveau du cinéma, que nous soyons forcés de réfléchir à notre mission ${ }^{59}$. "Ce n'est pas le lieu d'évoquer ici plus amplement le très sévère diagnostic que pose Garand sur l'ONF

57. Studio E, Le Cinéma régional de la production française (juin 1982), 12. Le texte en italique est souligné dans le document original.

58. Le Rapport du Comité d'étude de la politique culturelle fédérale est remis en novembre 1982. On y recommande, entre autres, la transformation de l'ONF en un "centre de recherche et de formation artistique et scientifique pour la production de films et de vidéos », ce qui veut dire concrètement l'abolition de l'ONF tel qu'il existe. Les réactions négatives ne se limitent pas au personnel de l'ONF. Partout au Canada, des utilisateurs de films de l'ONF lui manifestent un appui massif. La campagne pour le maintien de l'ONF déborde à l'étranger. Gilles Carle dira de ce rapport : “C’est un travail triste, sournois et qui prend ombrage de la réalité en ayant l'air de regarder le soleil. " «La vidéo et la mort», dans Carol Faucher, dir., La production française à l'ONF. 25 ans en perspectives (Cinémathèque québécoise, 1984). Certains éléments du rapport se retrouveront dans la Politique du film et de la vidéo qu'annonce le ministre des Communications Francis Fox le 29 mai 1984 (que l'ONF soit un «centre mondial d'excellence en matière de production de films et de vidéos" et un "centre national de formation et de recherche dans l'art et la technique du film et de la vidéo»). Toutefois, à la suite de l'élection de l'été 1984 qui porte Brian Mulroney au pouvoir, son application et son adaptation reviendront au nouveau titulaire du poste Marcel Masse. Voir aussi sur le sujet Gary Evans, (1989), 286-95.

59. L'Heure des choix (6 décembre 1982), 1. 
et les choix tragiques qu'il envisage, mais remarquons qu'il ne parle jamais des régions de manière spécifique; le drame est global à ses yeux. La situation générale de la Production française ne s'améliore pas. Le 14 juin 1983, Garand envoie à François Macerola un mémo intitulé Un ultime cri d'alarme pour parler d'une situation financière 1983-1984 qui s'annonce pire que la précédente et qui met en danger la section française et sa production. Entre temps, tous les studios doivent subir de nouvelles coupures, dont $600000 \$$ pour les régions. Parallèlement, dans leur planification 1983-1984, celles-ci constatent qu'elles doivent se rendre incontournables aux yeux du quartier général par la production d'un cinéma nécessaire, voulu, innovateur et visible. Les moyens spécifiques qu'elles se donnent tiennent donc à la qualité du cinéma à produire et à sa visibilité ${ }^{60}$.

Après quelques années de programmation sous l'empire du nouveau mode de fonctionnement mis en place au début de la décennie, le Studio E fait le bilan de sa programmation et de ses relations avec le Comité du programme et propose, en mai 1984, au nouveau directeur de la Production française, Daniel Pinard, un projet pilote de programmation sectorielle découlant du texte de réflexion Programmer le cinéma régional diffusé quelques jours plus tôt. Essentiellement, ce projet pilote d'un an, conçu comme une expérience particulière pour un secteur particulier, vise à pallier les carences du processus de programmation, où le Comité du programme et la direction de la Production française n'ont jamais pu trouver le moyen d'intégrer dans leurs structures la représentativité de l'élément francophone hors Québec. À sa réunion du printemps de 1984, le CA approuve à nouveau les buts et objectifs du Programme régional français qui permet à l'ONF de s'implanter partout au Canada dans ce qui est son territoire culturel et matériel. On comprend de tout ce contexte que les régions se sentent toujours en période transitoire depuis la restructuration et, notamment, qu'elles sentent le besoin d'affirmer leur spécificité, car les cinéastes de Montréal n'acceptent toujours pas la place qu'elles occupent.

Mais l'ONF ne va pas s'embarquer dans ces changements ponctuels puisqu'il s'attaque à l'époque à un plus gros morceau, le nouveau Plan d'exploitation quinquennal (adopté à l'automne de 1984) où l'importance des régions est réaffirmée. On sait, dans le cas de la régionalisation, qu'il s’agit d'un objectif politique canadien et, de ce point de vue, il importe peu que

60. Les studios régionaux vont même publier à partir de juin 1984 et jusqu'en décembre 1986 une revue, Le Cinéma des autres (au total cinq numéros), pour refléter leurs activités et celles de leurs régions. 
cette pratique ait encore de la difficulté à bien s'intégrer à la vie de l'ONF et peut-être même aux exigences complexes de la production cinématographique. Comme le constate le vétéran réalisateur Colin Low, l'ONF, à l'instar du Canada, est partagé entre la centralisation et la régionalisation ${ }^{61}$. Selon lui, pour que la régionalisation puisse être fonctionnelle, il faut lui accorder les ressources nécessaires. Or en période de restrictions, tel n'est pas le cas. À l’instar du vétéran réalisateur, plusieurs cinéastes pensent que l'ONF effectue un choix néfaste en coupant dans la mission nationale pour poursuivre la décentralisation et remplir l'objectif du plan : accroître la capacité de production des centres régionaux. À leurs yeux, il faut un centre fort pour que les régions produisent des œuvres de qualité, à la hauteur des normes de l'institution ${ }^{62}$. Mais si on admet que les pays comme le Canada ont besoin d'une intervention de l'État et d'une production publique pour développer une production nationale, il faut se demander quel est le rôle d'un organisme d'État œuvrant en production cinématographique dans une perspective nationale. Le rapport Applebaum-Hébert et surtout la Politique nationale du film et de la vidéo traduisent une volonté claire de l'État quant au développement cinématographique et à la répartition des pouvoirs et des mandats. Cela naturellement ne fait pas l'affaire des cinéastes ni même de la direction dans son ensemble. Ils ne pourront qu'essayer de tirer leur épingle du jeu et de s'adapter sans transformer trop radicalement les choses.

Le Studio E tente de son côté de profiter des circonstances pour pousser sur un ajustement de mandat par la publication d'un document de travail dont le titre en indique le sens: Pour une prise de possession du territoire culturel (et matériel) francophone à l'extérieur du Québec ${ }^{63}$. En février 1985, Daniel Pinard rencontre les producteurs régionaux pour répondre à leur proposition et surtout exposer le contexte. Il a le mandat de procéder à la réorganisation du Programme par la réduction des effectifs et des frais d'exploitation. Par conséquent, les régions seront mises à contribution. Il leur alloue $15 \%$ du budget du Programme français. Mais il veut en contrepartie une réduction de l'infrastructure régionale et refuse par conséquent que les régions prennent de l'ampleur. Il fait aussi sentir qu'il connaît les griefs des employés permanents contre les régions qui s'expriment dans le

61. La Minute de vérité. Réflexions sur un plan quinquennal (1984), 15. Low est une des figures importantes de l'ONF, y étant entré en 1945.

62. Raymond Gauthier, Programmer le cinéma régional. Note de service aux producteurs du studio $E$, (15 mai 1984), 8 .

63. 6 décembre 1984. Le texte reçoit un complément le 18 janvier 1985. 
rapport de février 1985 de Guy L. Coté : «Sans pour autant nier la légitimité pour l'Office de maintenir une production à saveur régionale, il serait assurément approprié de revoir, dans le contexte des années 80 , les modalités au moyen desquelles cet objectif pourrait être poursuivi. [...] Il faudrait aujourd'hui examiner si les objectifs de développement d'identité régionale par le cinéma, initiés par des fonds fédéraux, ne devraient pas dorénavant être poursuivis par l'ONF/NFB avec la collaboration financière des administrations provinciales, laissant à l'État fédéral la mission idéologique de refléter à travers l'Office les préoccupations nationales et la représentation internationale en matière de cinéma culturel et de service public ${ }^{64}$.» On perçoit bien la forte orientation du raisonnement, voulant, d'une part, lier la production régionale aux interventions provinciales (avec comme corollaire tacite qu'en l'absence de celles-ci, la production régionale devrait être réduite), et, d'autre part, suggérer que seul le cinéma des cinéastes québécois onéfiens refléterait, lui, les préoccupations nationales et remplirait une mission nationale (alors qu'on sait que ce cinéma ne s'intéresse souvent à rien d'autre que de québécois...). On ne peut pas dire que la bonne entente règne entre Montréal et les régions. On peut même penser qu'il s'agit là d'un état de fait normal, dans la mesure où le plan d'exploitation de 1984 prévoit restreindre la dimension du gâteau à partager.

\section{NOUVELLE STRUCTURE, NOUVEAU PLAN DE FONCTIONNEMENT}

Au moment où l'ONF se restructure et que la Production française devient le Programme français ${ }^{65}$, la discussion du projet de plan quinquennal va mobiliser les cinéastes pendant plusieurs mois. Mais leur discours n'est pas celui de l'institution. Sur le terrain des effectifs, l'objectif de celle-ci est clair : il faut arriver à un rapport $70 \%$ de pigistes $/ 30 \%$ de permanents (tous métiers confondus). On estime que l'impartition permettra d'amener du sang neuf, tout en réduisant les frais fixes. Même si on peut penser qu'en tant qu'organisme de savoir, l'institution devrait être sensible au discours de ses créateurs, elle ne peut définir ses politiques qu'en fonction des politiques plus générales de l’État. Après des mois de discussion, l'ONF publie son plan quinquennal. Un passage est consacré à la régionalisation. On y lit notamment : «L'Office mettra à la disposition des cinéastes francophones hors Québec des fonds de production qui seront attribués exclusivement au

64. Op. cit. (sommaire), iii. Le texte en italique est souligné dans le document original.

65. Cette réforme administrative, qui devient effective en 1985 sous la houlette du commissaire François Macerola, vise à gérer de manière intégrée production et mise en marché pour qu'il y ait complémentarité entre les différents intervenants. 
programme de régionalisation. Ces sommes d'argent seront établies en fonction de la proportion de francophones d'une région hors Québec par rapport à l'ensemble de la population française au pays. Parce que ces fonds sont de toute évidence insuffisants, nous devons nous associer en production avec d'autres organismes publics et parapublics. [...] Une réduction importante de l'infrastructure de production hors Québec nous permettra de réaliser des économies appréciables et d’augmenter par conséquent les montants alloués à la production proprement dite. Toute la production française hors Québec se fera à partir de deux centres de production, l'un à Moncton et l'autre à Toronto ${ }^{66}$."

Ce plan cause quelques remous parmi les régions, en Ontario notamment. Le producteur Paul Lapointe se déclare incapable d'assumer les responsabilités que le plan quinquennal voudrait lui confier, notamment parce qu'il estime ne pas pouvoir couvrir tout le territoire à l'ouest du Québec ${ }^{67}$. De plus, il croit que si le financement des activités doit être établi selon le rapport démographique des francophones hors Québec à l'ensemble francophone canadien, le même principe devrait s'appliquer à la répartition des fonds entre les régions. Belle bataille en perspective. Il estime en outre que seule l'Ontario jouit d'une situation lui permettant d'intégrer de façon significative à ses activités de production les ressources extérieures comme le souhaite le Plan. Celui-ci n'est pas sitôt adopté que Lapointe propose, le 28 novembre, la mise sur pied d'une seule unité, Programmation hors Québec, divisée en trois régions (Ouest, Ontario, Acadie) et dont le chef verrait aux activités de production, de mise en marché et de distribution et, par voie de conséquence, à la redistribution des sommes allouées. En fait, il recrée le Studio E. Le nouveau directeur du Programme français, Georges Dufaux, annonce ses recommandations, notamment que l'ONF respecte les différences régionales et tienne compte de leurs disparités en maintenant trois régions : l'Est, l'Ontario et l'Ouest sous la responsabilité d'un même studio, le Studio 3. Après une période de flottement, on repart à zéro et on peut dire que le cas des régions est pratiquement réglé. La décentralisation est là pour demeurer. Les régions connaissent un certain bouleversement dans la mesure surtout où, à la fin des années 1980, on cesse de produire dans l'Ouest parce qu'il n'y a pas de structures de production dans les milieux francophones. On fusionne l'Ontario et l'Ouest. Le bud-

66. Dispositions du plan d'exploitation relatif aux stratégies administratives à suivre en matière de production et de distribution à l'Office national du film du Canada (15 juillet 1985), 45.

67. Paul Lapointe, Programmation hors-Québec. Note de service à Daniel Pinard (28 novembre 1985), 8. 
get des deux régions est alors de 299500 \$ pour l’Acadie et de $163970 \$$ pour l'Ontario-Ouest, ce qui semble suffisant pour mettre en pratique le mot d'ordre du directeur du Programme français Robert Forget, en poste depuis 1989 : «viabilité».

Le studio des régions connaît une nouvelle réorientation au début des années 1990. On change encore son nom en Studio R et on le nomme «studio des minorités francophones». La ligne directrice qu'avance la Planification ${ }^{68}$, se résume maintenant en un mot : reflet. Reflet de la vitalité francophone, politique de reflet qui est au cœur du mandat national de l'ONF, reflet qui consolide les multiples identités régionales dans une identité nationale, reflet qui permet de donner la parole à ces minorités dans un moment privilégié d'osmose entre un milieu donné et un cinéaste créateur. Cela correspond tout à fait à l'objectif de visibilité que Michel Lacombe, le producteur du Studio, énonce dans sa planification d'avril 1994, tout comme il souhaite accroître le partenariat avec le privé afin de multiplier le nombre des productions. Si la région Ontario réussit bien dans ce domaine grâce à la vitalité de son économie cinématographique, à l'éventail des coproducteurs disponibles et à l'action de TVO, tel n'est pas le cas de l'Acadie où, au cours des deux dernières années, le studio a éprouvé de la difficulté à épuiser ses fonds, pourtant limités, à la suite de la panne sèche traversée par son seul partenaire privé, les Productions du Phare-Est. C'est dans ce contexte que le nouveau producteur acadien, Pierre Bernier, avancera qu'il faut adapter l'ONF à la région et non pas la région à l'ONF. Il réussit même à faire admettre qu'un représentant des régions soit membre du Comité du programme de manière statutaire. Parallèlement, il encourage les cinéastes à se prendre en main et à se donner des outils qui les rendraient un peu plus indépendants de l'apport de l'ONF. À l'automne de 1994, un groupe de cinéastes et de vidéastes du Nouveau-Brunswick demande au gouvernement provincial d'établir une société de développement cinématographique à l'image de ce qui existe déjà en Nouvelle-Écosse.

L'ONF va appuyer la création de Film Nouveau-Brunswick parce que cet organisme devrait permettre aux cinéastes de la province de réaliser plus facilement des films qui leur ressemblent. Il estime aussi que plus il y aura de travail dans la région, plus les techniciens et les cinéastes pourront vivre en exerçant leur métier dans les Maritimes, ce qui aura pour conséquence d'encourager la réalisation sur place de toutes les étapes d'un 
film. C'est moins lourd de faire affaire avec un studio sur place que d'amener un réalisateur et des techniciens à Montréal. Cette volonté de développement des structures locales de production professionnelle arrive au moment où l'ONF traverse une autre rude période. Pierre Bernier y fait écho dans la section qu'il rédige de Planification 1995-1996: «Coupure, fermeture, étude, rapport Secor, Comité des sages, révision du mandat de l'ONF et j'en passe. [...] Vous comprendrez que dans un tel climat, il n'est pas facile ni pour vous, ni pour les cinéastes, ni pour les producteurs de regarder dans la boule de cristal qui est, soit dit en passant, quelque peu embrouillée ${ }^{69}$." En ces années qui suivent la Grande Crise de 1994, l'ONF décide d'abandonner la fiction pour privilégier l'animation et le documentaire, et dévoile un plan de restructuration, ONF 2000, duquel, incidemment, découlera la création des studios documentaires régionaux où s'affirme la volonté de faire du documentaire d'auteur. Telle est la situation qui prévaut jusqu'en 2001, alors que le nouveau commissaire, Jacques Bensimon, en poste depuis le 18 juin 2001, propose une réorientation de l'ONF dont le mandat spécifique pour les régions serait de mieux desservir leurs besoins ${ }^{70}$.

\section{CONCLUSION}

Cette histoire du cinéma régional fait ressortir une dimension de la production onéfienne francophone occultée par ceux qui l'écrivent toujours du point de vue de Montréal et de la problématique québécoise. Ces personnes, dont je fais partie à titre d'historien de l'ONF, doivent faire amende honorable et ajuster leur discours à la complexité de la réalité cinématographique de langue française au Canada. Il faut poser l'existence du cinéma régional depuis ses propres termes et en relation avec le reste du cinéma de la Production française et du Programme français. On découvre qu'il existe plus de deux solitudes au cour de l'ONF. Une étude plus approfondie pourrait permettre de rechercher et d'analyser plus systématiquement le discours régional dans le discours global de l'institution. Non pas pour nier l'une ou l'autre approche, mais pour mettre en lumière les différents discours culturels et nationaux qui coexistent et même s'opposent au sein d'un même organisme d'État qui est constitué de plusieurs instances interagissantes : conseil d'administration, haute direction, directions française et

69. "Vision, Révision et Prospective du Studio de l'Acadie», dans Programme français, Planification 1995-1996, (avril 1995), 99.

70. Impact 2001-2006 (août 2001), 5. 
anglaise, deux Comités du programme, Atelier des cinéastes, syndicat. Les pages qui précèdent démontrent, croyons-nous, la nécessité de voir l'histoire de la production francophone de l'ONF sous un autre angle que celui du discours historiographique dominant. Elles ouvrent la voie à une analyse évolutive de la dimension régionale dans le fonctionnement de l'organisme et dans les sujets que les films abordent. Elles attirent l'attention sur la prise en charge, dans l'analyse du champ culturel, du contexte que constituent les luttes nationalistes et politiques qui marquent les relations entre le Québec et la francophonie hors Québec, et entre le gouvernement fédéral et les régions du Canada. Elles ne forment pas une conclusion mais une invitation : une invitation à la découverte, à la remise en question et à l'approfondissement de la réflexion. L'histoire de l'ONF représente un chapitre particulier dans l'histoire de la régionalisation au Canada et de la dynamique centre/région. L'écrivain et cinéaste acadien Herménégilde Chiasson a déjà déclaré : «Le Québec a toujours constitué un élément perturbateur dans la conscience acadienne ${ }^{71}$.» Le présent texte aura montré que le cinéma régional constitue également un élément perturbateur dans la conscience onéfienne et dans l'entendement qu'on se fait de l'histoire de l'institution. 